\title{
SERPINA1 is a direct estrogen receptor target gene and a predictor of survival in breast cancer patients
}

\author{
Hei Jason Chan ${ }^{1}$, Haiqing $\mathrm{Li}^{2}$, Zheng Liu ${ }^{3}$, Yate-Ching Yuan ${ }^{3}$, Joanne Mortimer ${ }^{4}$, \\ Shiuan Chen ${ }^{1}$ \\ ${ }^{1}$ Department of Cancer Biology, Beckman Research Institute of the City of Hope, Duarte, California, USA \\ ${ }^{2}$ Department of Molecular Medicine, Beckman Research Institute of the City of Hope, Duarte, California, USA \\ ${ }^{3}$ Bioinformatics Core Facility, Beckman Research Institute of the City of Hope, Duarte, California, USA \\ ${ }^{4}$ Department of Medical Oncology, Beckman Research Institute of the City of Hope, Duarte, California, USA \\ Correspondence to: \\ Shiuan Chen, e-mail: schen@coh.org \\ Keywords: estrogen receptor, breast cancer, endocrine resistance, SERPINA 1, survival analysis \\ Received:December 19, $2014 \quad$ Accepted: June 19,2015 Published: June 29, 2015
}

\section{ABSTRACT}

Of all breast cancer patients, about $70 \%$ are ER+ and $10 \%$ are ER+/HER2+. The ER+/HER2+ patients have a worse outcome compared to ER+/HER2- patients. Currently there is a lack of effective prognosis biomarkers for the prediction of outcome in ER+/HER2+ patients. Genome-wide differences in ER binding between the endocrine-responsive and endocrine-resistant cells were discovered using ChIPseq, and combined with gene expression microarray data to identify direct ER target genes. These genes were correlated to survival outcome using publicly available breast cancer patient cohorts. We found the expression of the gene SERPINA1 to have a significant predictive value for the overall survival (OS) of ER+ patients in the TCGA cohort, and validated this finding in the Curtis cohort. SERPINA1 also has a significant predictive value for the OS of ER+/HER2+ patients in the TCGA cohort, with validation in the Bild cohort. The expression of SERPINA1 can be suppressed by fulvestrant and HER2 sIRNA. Our results indicate that ER is constitutively activated, resulting in an E2-independent ER binding to the SERPINA1 gene and upregulation of SERPINA1 expression. Importantly, results of survival correlation suggests that high expression of SERPINA1 could be predictive for a better clinical outcome of ER+ and ER+/HER2+ patients.

\section{INTRODUCTION}

The estrogen receptor $\alpha$ (ER) is a crucial transcription factor that is required for cell proliferation in the majority of breast cancer cases, which accounts for about $70 \%$ of all breast cancers. A major treatment of ER+ breast cancer is endocrine therapy using anti-estrogens like tamoxifen or aromatase inhibitors (AIs). However, a significant number of $\mathrm{ER}+$ patients are not responsive to such treatment (i.e., de novo resistance) and some patients develop resistance during endocrine therapy (i.e., acquired resistance). Previous studies in our lab have shown that the ER is required for growth in both endocrine (therapy)responsive and endocrine-resistant breast cancer cells, but only endocrine-responsive cells require estrogen for the proliferation [1]. The global genomic binding profile of
ER has been well documented in endocrine-responsive breast cancer cells but not in endocrine-resistant cells [2]. To investigate the molecular action of AIs, our laboratory has generated an aromatase-overexpressing MCF-7 cell line, i.e., MCF-7aro [3]. For this project, we used MCF7 aro cells as a model for endocrine-responsive breast cancer, and Long Term Estrogen Deprived (LTEDaro) cells as a model for endocrine-resistant breast cancer [4]. In the endocrine-responsive breast cancer cells, $17 \beta$-estradiol (E2) acts as a ligand and binds to ER, activating the ER and causing its translocation from the cytosol to the nucleus. The E2-bound ER then binds to the chromatin to regulate the expression of target genes. In the endocrine-resistant cells, the ER can be activated by other mechanisms such as phosphorylation, so even in the absence of E2, the ER is able to bind to chromatin and 
activate target genes. The ligand-independent activation of ER is thought to play key roles in endocrine-resistant breast cancer because the ER degrader, fulvestrant (ICI $182,780)$, is able to suppress the expression of ERregulated genes [5]. The goal of this study is to find such ER binding sites and target genes which will improve our understanding of the roles of ER in both endocrineresponsive and resistant cancers.

To better understand the physiological action of ER-target genes, we used chromatin immunoprecipitation with next-generation sequencing (ChIP-seq) as a tool to identify differences in ER binding between endocrineresponsive and endocrine-resistant cell lines in a genomewide manner. In previous studies, our lab has performed Affymetrix GeneChip genome-wide microarray gene expression analysis to detect differentially estrogenregulated genes [1], but these target genes include direct and also indirect ER targets. The combination of ChIPseq with microarray gene expression analyses allows us to identify the direct ER target genes. We had hypothesized that the identification of such genes could allow us to find a gene that acts as a biomarker associated with endocrine response of breast cancer, which would be valuable for the prediction of the consequence of endocrine therapy and could facilitate the selection of the most effective treatment options for patients. Our extensive ER ChIPseq analysis has resulted in a candidate gene SERPINAI that has a clear ER binding site in its promoter region and higher expression level in LTEDaro DMSO (i.e., in the absence of E2). The levels of SERPINA1 mRNA have been found to be significantly higher in LTEDaro DMSO than in E2-treated MCF-7aro E2 [1]. Based on our survival analysis results using the publicly available large panel of The Cancer Genome Atlas (TCGA) 779 breast cancer patient cohort [6] with clincopathological information, we further hypothesize that the upregulation of SERPINA1 in endocrine-resistant cells requires HER2 and has significant association with better survival outcome for ER+/HER2+ breast cancer.

SERPINA1, also known as $\alpha 1$-AntiTrypsin (AAT), is a protease inhibitor that can act on a variety of targets such as serine proteases. It has been demonstrated that SERPINA1 expression can be stimulated by E2 in MCF-7 cells, and high expression of this protein inhibits colony formation [7]. SERPINA1 has been proposed as a biomarker for various diseases such as Cutaneous Squamous Cell Carcinoma [8], Hepatitis B [9], insulinomas [10], NSCLC [11], papillary thyroid carcinoma [12] lung cancer [13] and breast carcinoma [14-16]. Unexpectedly, our results allow us to hypothesize that the single gene SERPINA1 is a significant predictor of survival in ER+ and ER+/HER2+ breast cancer patients. Patients with ER+/HER2+ breast cancer generally have a worse outcome compared to ER+/HER2- patients $[17,18]$. Currently there is no known predictive marker for the treatment outcome of ER+/HER2+ breast cancers [19], thus the ability of SERPINA1 to predict the survival of this intrinsic subtype of breast cancer patients is valuable.

\section{RESULTS}

\section{Bioinformatics analysis of ER ChIP-seq and microarray expression data}

The ER binding sites were annotated with the genes, and we plotted the number of binding sites against the distance to the closest transcription start sites (TSS). Comparison of the number of ER binding sites close to the TSS demonstrated that the distribution of the number of binding sites in the LTEDaro DMSO was comparable to that found in the MCF7aro E2 (Figure 1A). This confirms that the ER binding in $\mathrm{MCF}-7$ aro is dependent on estrogens as expected, and most importantly, significant ER binding can occur without any hormones in LTEDaro cells. Analysis of the correlation between the number of binding sites and binding intensities also demonstrated that both LTEDaro DMSO and MCF-7aro E2 had a comparable normal distribution (Figure 1B).

Based on the overlap analysis of ER binding sites described in Materials and Methods section, we performed a comparison between ER binding sites in hormoneindependent LTEDaro DMSO and hormone-dependent MCF-7aro E2 cells, as shown in Figure 1C. A majority of the binding sites were in the common group, but it should be emphasized that although the common sites shared the same location, the ER binding intensities were not always similar between LTEDaro DMSO and MCF-7aro E2.

Figure 2A shows a comprehensive analysis workflow to determine the 350 differentially regulated genes annotated for further validation using large patient cohorts with survival information.

\section{Survival analysis using publicly available breast cancer patient cohorts}

To determine the physiological significance of ERbinding genes in endocrine resistant cells, the resulting list of 350 genes was further analyzed for the ability to predict patient survival using the TCGA breast cancer patient cohort. 2-means clustering was adopted to cluster patients into high and low risk subgroups based on the 350 genes. As a whole, the group of 350 genes did not have a significant predictive value (data not shown). As described in the Materials and Methods section, using a Cox score cutoff of 2.39, the panel of 35 genes was further filtered for better survival correlation.

These 35 genes were then inspected individually in the IGV genome browser for ER binding site quality in order to narrow down the candidates for further qPCR validation and survival analysis. As described above, we are interested in ER binding sites that are dependent on hormones in the MCF-7aro cells but have significant ER binding in the LTEDaro DMSO cells. During the visual 
A

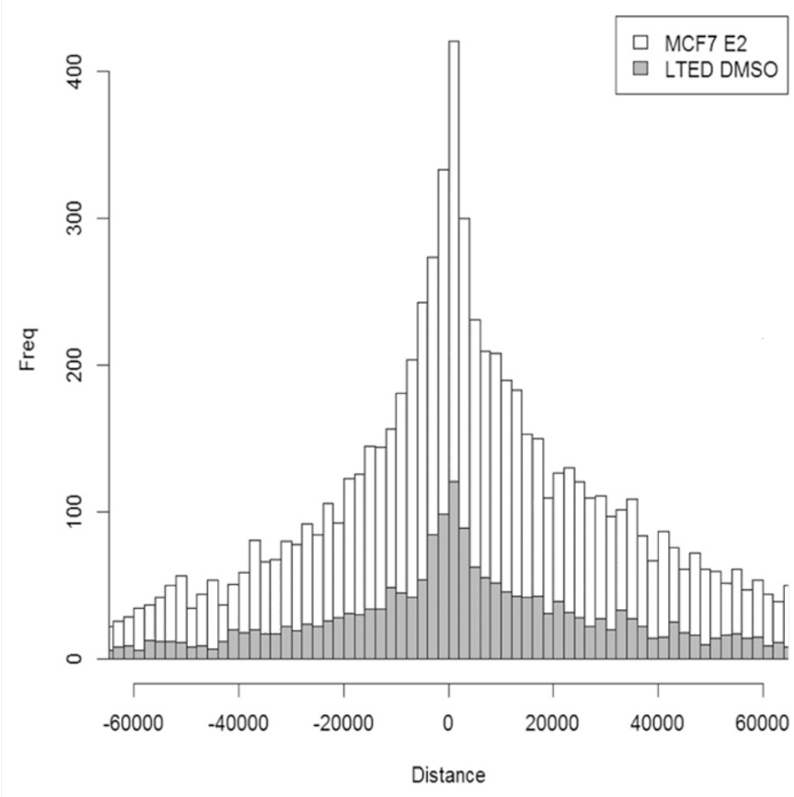

B

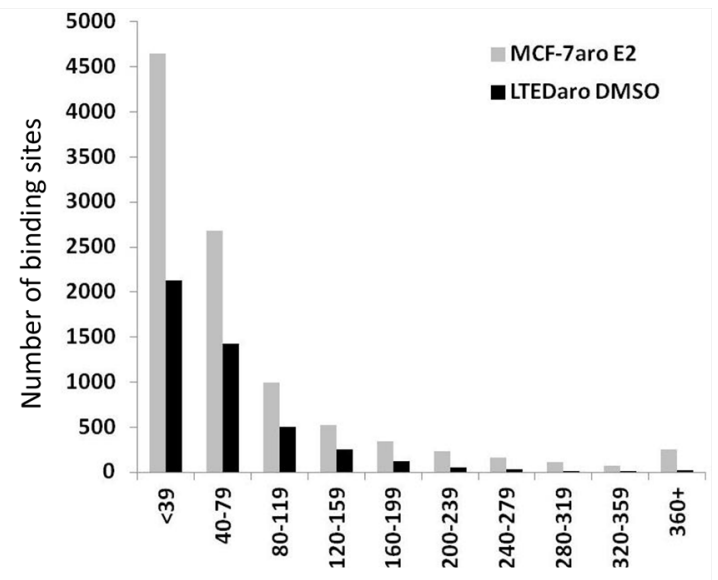

C

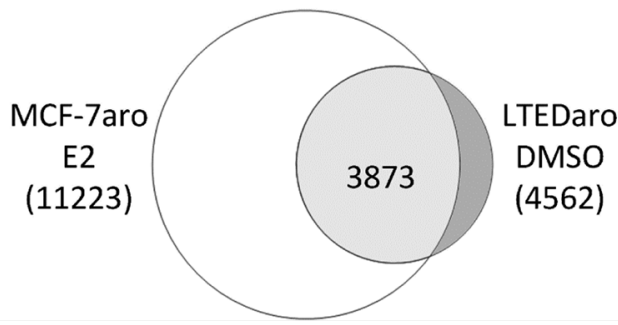

Figure 1: ER is able to bind chromatin independently of E2 in LTEDaro cells. A. Distribution of ER binding sites relative to the closest TSS. MCF-7aro DMSO binding sites are almost evenly distributed and MCF-7aro E2 binding sites are more abundant close to the TSS. LTEDaro DMSO binding sites are abundant close to the TSS with a similar trend as the MCF-7aro E2. B. Correlation between the number of binding sites and binding intensities of LTEDaro DMSO and MCF-7aro E2. C. Comparison of LTEDaro DMSO and MCF-7aro E2 shows a majority of ER binding sites occur at the same location, although the intensities may be different.

inspection we took into account the overall intensity of ER binding, the distance of the binding from the TSS, the ratios of MCF-7aro E2 to MCF-7aro DMSO binding, and ratios of LTEDaro DMSO to MCF-7aro E2 binding. Based on these criteria, we selected 3 genes with negative Cox score and 8 genes with positive Cox score from the panel of 35 genes. The 11 genes were then correlated with survival in ER+ and ER- patients using Kaplan Meier survival analysis. Based on $p$-values and biological relevance, we decided to focus on our analysis on the best candidate with strong ER binding in LTEDaro DMSO, the SERPINA1 gene from the negative Cox score group. SERPINA1 was brought to our attention because it has been reported to be an ERregulated gene in breast cancer cells [29]. In our analysis, SERPINA1 has a well-defined ER binding site with the distinctive property that ER binding in LTEDaro DMSO was found to be stronger than MCF-7aro E2 (Figure 2B). According to our previous Affymetrix microarray data, the expression level of this gene was about 3.4 fold higher in LTEDaro DMSO compared to MCF-7aro E2, and this difference is significantly higher according to our qPCR analysis (Figure 4A). Further literature search reveals that SERPINA1 is a known marker for good prognosis in cancer $[12,14]$. As indicated by a negative Cox score, a higher expression of SERPINA1 was found to associate with better patient survival outcome in the TCGA large patient cohort, and validated using the Curtis and Bild breast cancer patient cohorts. These results will be discussed in more detail in the Kaplan Meier analyses. Before we performed the detailed survival analyses, we confirmed the SERPINA1 ER binding and gene expression regulation by ER and HER2.

\section{The promoter of SERPINA1 has an ER binding site}

Although E2 was reported to up regulate the expression of SERPINA1 twenty years ago [7], the mechanism was unknown at that time. A direct ERmediated regulation of its expression in MCF-7 cells was reported by Simpson et al. [29], and it was reported that E2 addition did not significantly enhance the ER binding to the ERE in the promoter of SERPINA1. Our ER ChIPseq analysis confirms that the SERPINA1 gene has an ER binding site within the promoter region which overlaps with the TSS (Figure 2B), and the full ERE motif was found within this binding site by mapping known motifs (Supplementary Figure 1), which agrees with the previous study by Simpson et al [29]. In the MCF-7aro cells, the binding of ER to this site is dependent on estrogen, but in the LTEDaro DMSO cells, the ER binding has a higher intensity than MCF-7aro E2 even without estrogen. The ChIP PCR validation confirms the binding in LTEDaro DMSO (Figure 2C). 
A

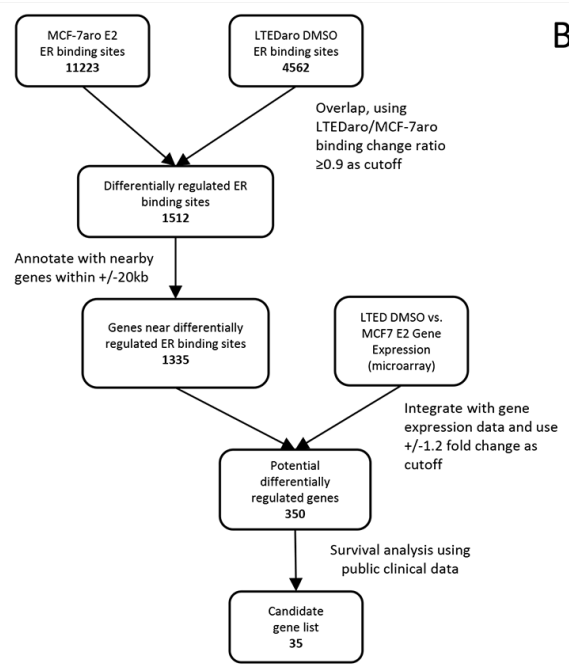

B

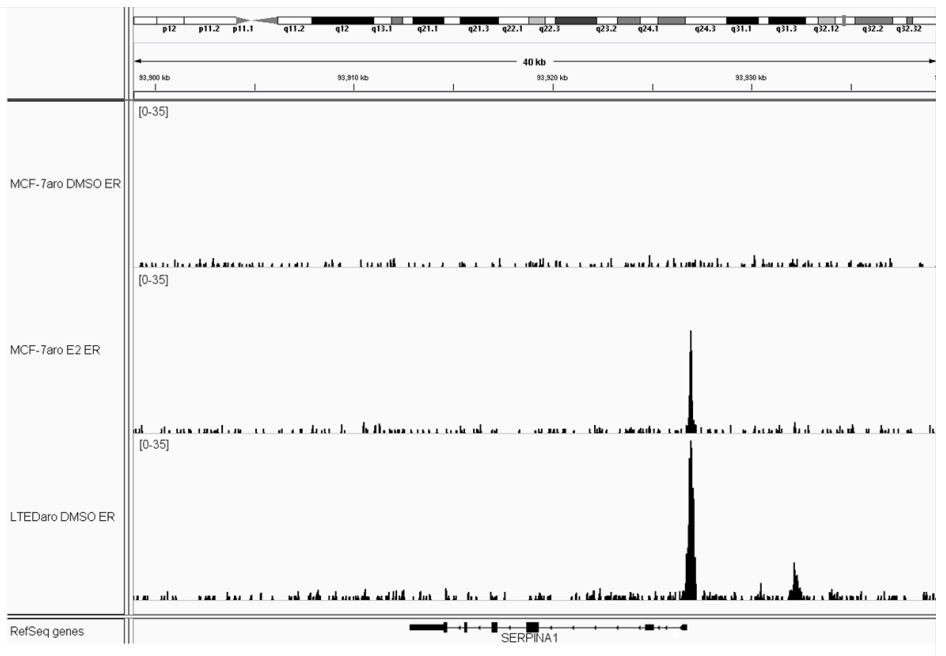

C

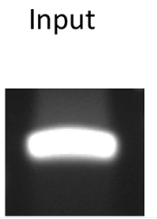

LTEDaro DMSO

$\operatorname{lgG}$

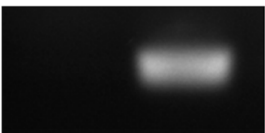

Figure 2: Identification of SERPINA1 as an ER target gene with a distinctive ER binding site in the promoter region. A. Summary of bioinformatics analysis of Illumina ChIP-seq and Affymetrix GeneChip gene expression microarray data, leading to the discovery of SERPINA1 and its potential regulation by ER and HER2. B. SERPINA1 has an ER binding site proximal to the TSS with higher level of ER binding in the LTEDaro DMSO cells compared to MCF-7aro E2 cells. In contrast, other well-known ER target genes have higher level of ER binding in the MCF-7aro E2 cells instead. C. ChIP PCR validation of ER binding site proximal to the TSS of SERPINA1 confirms the ER binding in LTEDaro, as detected by ChIP-seq.

\section{SERPINA1 promoter is $\mathrm{E} 2$ responsive}

To validate the activity and E2 response of the SERPINA1 promoter, we cloned the full length and deletion mutants as described in the Materials and Methods section (Figure 3A). From the ChIP-seq peak calling data, the ER binding site covers a region approximately $2 \mathrm{~kb}$ which overlaps with the TSS. We cloned the region that covers the TSS and upstream $2 \mathrm{~kb}$ region, with a total length of $2.1 \mathrm{~kb}$. This promoter construct treated with DMSO control showed a basal level of luciferase reporter activity compared to the empty vector control (Figure 3B). Upon E2 treatment, the luciferase activity increases, demonstrating that the full length promoter transcriptional activity is inducible by E2. In the truncated promoter fragments with and without the predicted ERE sequence, we observed a basal activity similar to the full length promoter treated with DMSO. With E2 treatment, we expected to see increased luciferase activity in the promoter fragment containing the ERE, but not in the ERE deleted fragment. Our results show that with E2 treatment, there is no significant increase in SERPINA1 expression in both constructs. This suggests that the proposed "ERE" alone is not sufficient, and there may be other sequences upstream of the predicted ERE that are needed for the E2 response.

\section{ER-dependent and HER2-dependent regulation of SERPINA1 expression in endocrine-responsive and -resistant cells}

In the MCF-7aro, LTEDaro and HER2-aro cell lines we examined, the SERPINAI expression is up regulated with E2 treatment and suppressed by the ER degrader, fulvestrant (ICI 182, 780) (Figure 4A). A search for SERPINA1 in the Gene Expression Omnibus (GEO) database provided support that SERPINA1 expression is stimulated by $\mathrm{E} 2$ in an ER-dependent manner [30, 31], and unexpectedly, by HER2 [32]. Since ER is known to be activated through ER-HER2 crosstalk in ER+/HER2+ breast cancer cells, we performed experiments to determine whether the expression of SERPINA1 could be regulated by HER2. The expression level of SERPINA1 in two tested HER2-overexpressing cell lines, HER2-aro [5] and LTEDaro cells [33], was found to be significantly higher than that in MCF-7aro cells, demonstrating that SERPINA1 is a HER2 regulated gene (Figure 4A). The HER2-dependent regulation of SERPINA1 expression was confirmed further by the down regulation of its expression by the treatment of siRNA targeting HER2 (Figure 4B). In attempt to further support our findings in additional cell lines, we checked the SERPINA1 expression level in BT474 and MDA-MB-361cells, which express both ER and HER2 endogenously. The results show that E2 and 
A

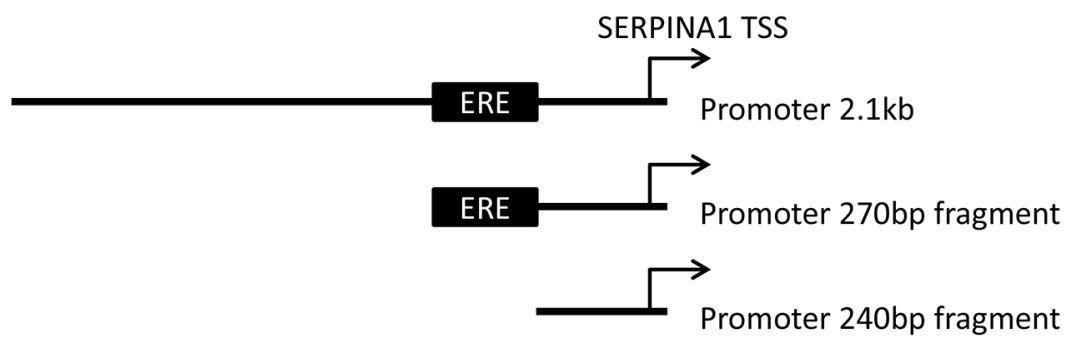

\section{B}
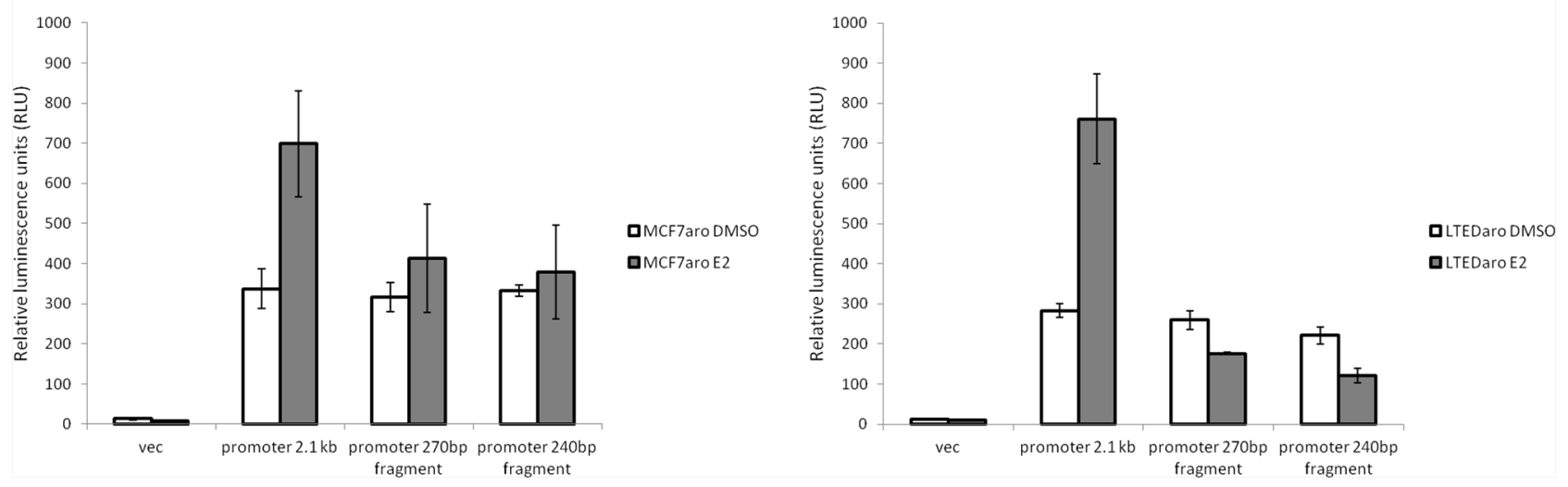

Figure 3: Cloning of SERPINA1 promoter and deletion mutants into luciferase reporter vectors and validation of activity. A. The SERPINA1 full length promoter and two deletion mutants, with and without ERE, were cloned into luciferase reporter vectors. B. Luciferase activity assays of the reporter constructs in MCF-7aro and LTEDaro cells with E2 treatment shows E2 responsive transcriptional activation in the full length promoter.

ICI treatment did not significantly change SERPINA1 levels, although there may be a weak induction by E2 in BT-474 cells (Supplementary Figure 2).

\section{Significance of SERPINA1 expression in ER+ and ER+/HER2+ breast cancer}

Based on our findings that the expression of SERPINA1 is regulated by both ER and HER2, we then performed the Kaplan Meier survival analysis by dividing the patients into high and low expression groups based on the median of the single gene SERPINA1. We found that in the TCGA training cohort with $570 \mathrm{ER}+$ patients, this gene showed a statistically significant predictive value $(p=0.0002)$ (Figure 5A). In contrast, the same analysis performed on the ER- patients was not statistically significant (Figure 5A), confirming that it is an ERregulated gene. We validated this finding in the Curtis cohort using Disease Free Survival (DFS) analysis with $986 \mathrm{ER}+$ patients $(p=0.01)$ (Figure 5B) which correlated with OS to serve as strong prognostic factor for patient treatment outcome.

To further validate our findings, we decided to carry out the survival analysis in four additional patient cohorts [24-28] (Table 1). However, we failed to observe any correlation between SERPINA1 levels and survival in other four cohorts of ER+ patients, namely Chin, Pawitan, Desmedt, Sotiriou (data not shown). Since we confirmed that the expression of SERPINA1 can also be regulated by HER2, we then checked the HER2 status of patients in the six cohorts and found that only the TCGA, Curtis and Bild cohorts had a significant number of HER2-positive patients in ER+ subcohorts, whereas the other 4 cohorts had mostly HER2-negative patients or patients with unknown HER2 status in their ER+ subcohorts (Table 1). Such observations led us to propose that the HER2 status is related to the predictive value of SERPINA1 on patient survival outcome. To verify this hypothesis, we performed further survival analysis with subgroups of patients by separating the patients based on ER and HER2 status. In the TCGA cohort, we subdivided the ER+ patients based on HER2 status, and found that the SERPINA1 has a significant predictive value in the ER+/HER2+ group with 82 patients $(p=0.045)$ but not the ER+/HER2(Figure 6A), ER-/HER2+, or ER-/HER2- patients (ERdata not shown). For validation we used the Bild breast cancer patient cohort with 61 ER+/HER2+ patients [24]. The $p$-value is 0.075 which was slightly above 0.05 , perhaps due to the low number of ER+/HER2+ patients (Table 1), but the trend of separation was observed visually (Figure 6B). We performed the same analysis on the Curtis ER+/HER2+ patients, and also observed a visual 

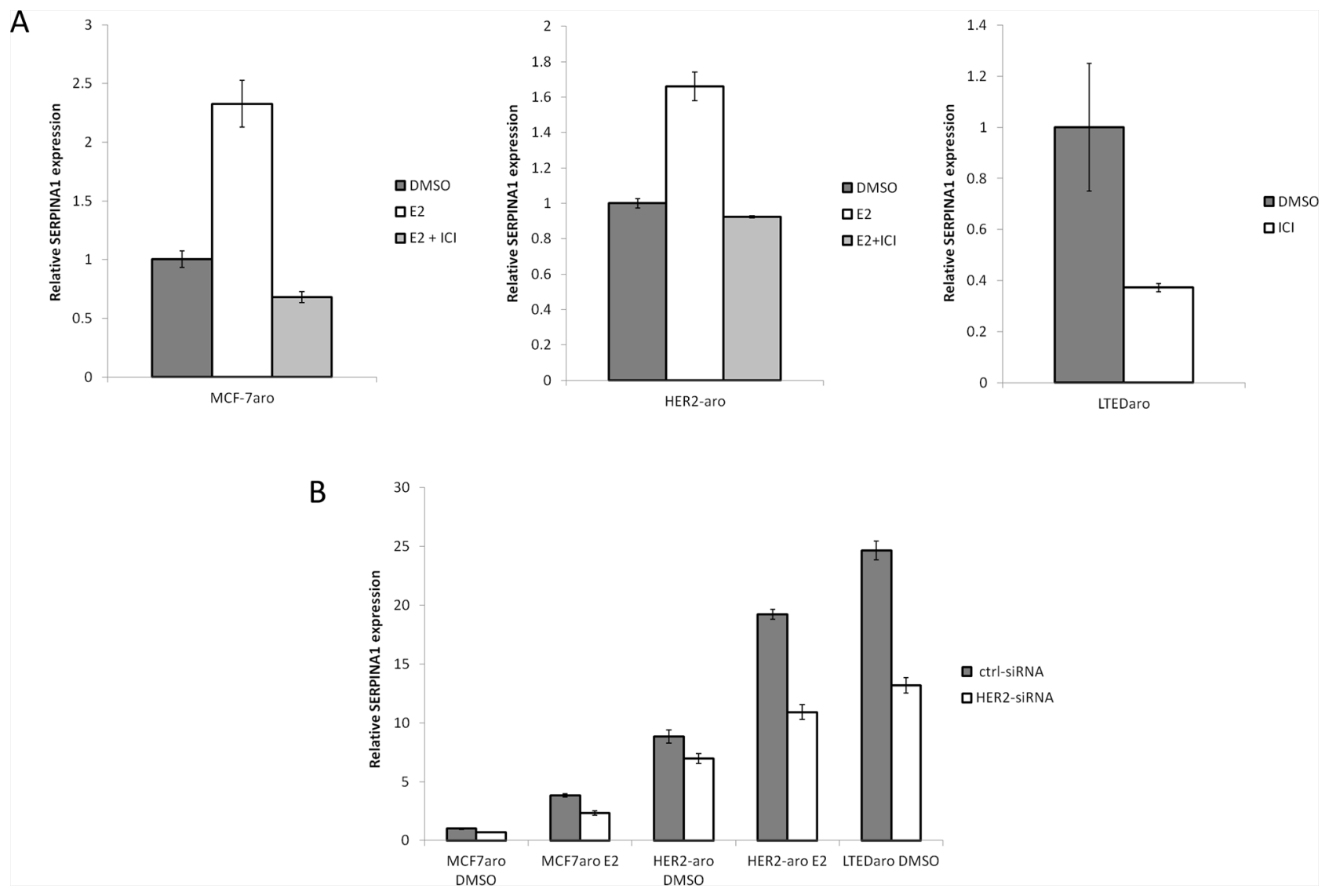

Figure 4: E2 and HER2 regulate the expression of SERPINA1 through the ER. A. Gene expression analysis of SERPINA1 by qPCR shows that SERPINA1 expression in MCF-7aro E2, HER2-aro E2, and LTEDaro DMSO can be suppressed by ICI treatment. B. Comparison of the SERPINA1 expression in the control-siRNA treated cells shows that HER2-aro and LTEDaro cells have a higher expression compared to MCF-7aro. siRNA knockdown of HER2 shows that SERPINA1 is downregulated by about $40 \%$ when HER2 levels are reduced.

separation of the two curves, but the curves intersected each other at the earlier timepoints, and the $p$-value was 0.14 , so these results were not statistically significant. To further establish the value and uniqueness of SERPINA1 as a predictive marker, we investigated the predictive ability of some well known ER target genes TFF1 (pS2), $P G R$ and GREB1. We performed the survival analyses by grouping the patients in the TCGA cohort based on ER status only, and both ER and HER2 status (Supplementary Figure 3). In ER+ and ER+/HER2+ patients, the 3 genes were not able to separate the patients into high and low risk groups. This further supports the unique ability of SERPINA1 to predict patient survival, because SERPINA1 is regulated by both ER and HER2.

\section{DISCUSSION}

ER is a key player in estrogen (or hormone)dependent breast cancer, and its action can be modified through many mechanisms (see a recent review by Manavathi et al. 2013) [34]. Ross-Innes et al [35] provided an excellent example to show that changes in ER binding is associated with clinical outcome in breast cancer. There have been extensive studies of ER binding in estrogenresponsive cells/tissue through ChIP-on-chip and ER ChIP-seq analyses [30, 36-39]. From our Illumina ChIPseq and Affymetrix GeneChip microarray data, it is clear that the ER in the LTEDaro cells behaved differently from that in the MCF-7aro cells. The analysis of distance to transcription start site (TSS) showed that in the estrogenresponsive MCF-7aro cells, the ER recruitment proximal to the TSS was dependent on E2, but in the resistant cells the ER recruitment to the same region could occur without E2. Comparison of the intensity and number of peaks revealed that in the MCF-7aro cells most of the ER binding were very weak without E2, and the ER binding was greatly enhanced when E2 was present. On the other hand, in the LTEDaro DMSO cells, a significant number of ER binding sites could be detected. Our ER binding data supports previous proliferation studies generated from this and other laboratories [40]. In the MCF-7aro cells, proliferation is entirely dependent on the E2-mediated activation of ER, and other growth factor pathways are not essential for the proliferation of these cells. LTEDaro cells are still dependent on the ER pathway for proliferation, as indicated by the fact that fulvestrant (ICI 182, 780) was 


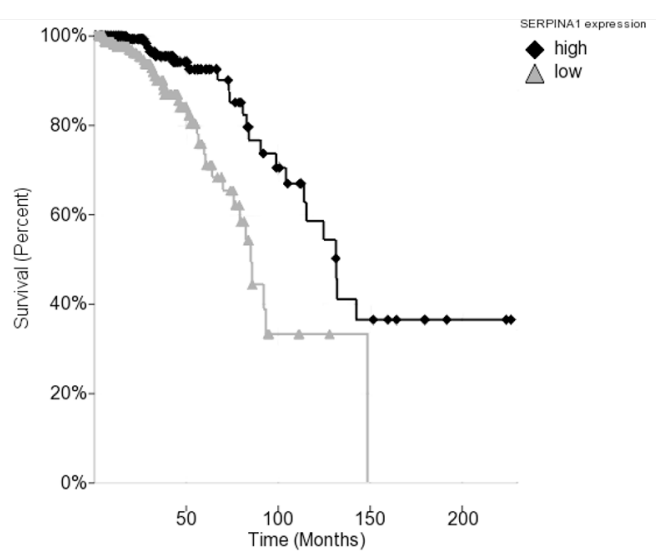

ER+ patients $(n=570, p=0.0002)$

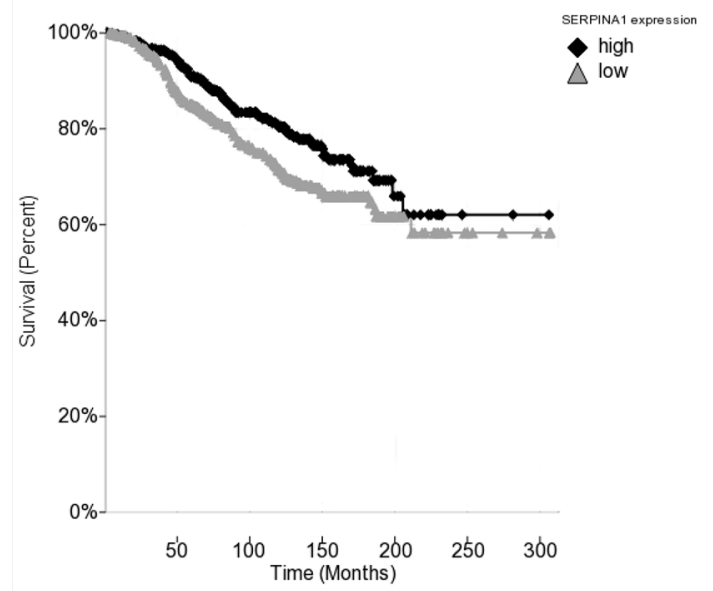

ER+ patients $(n=986, p=0.010)$

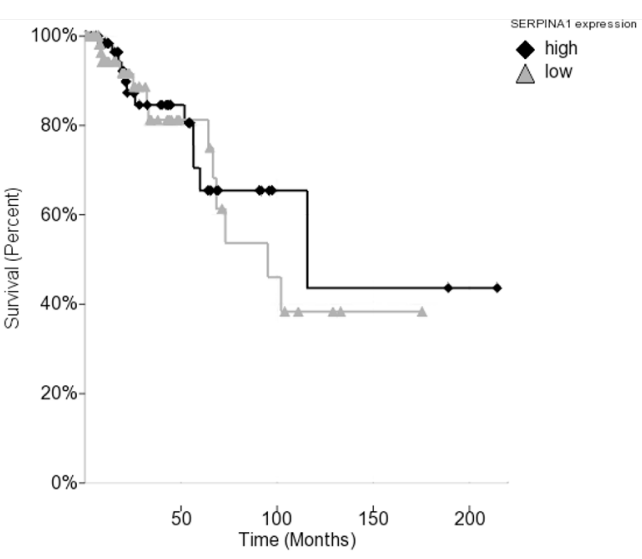

ER- patients $(n=172, p=0.66)$

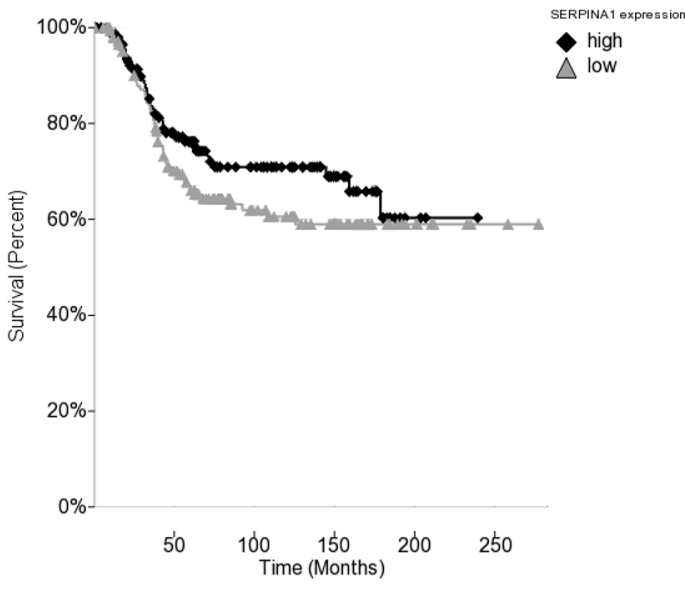

ER-patients $(n=298, p=0.20)$

Figure 5: SERPINA1 expression level is a predictive marker for ER+ breast cancer patient survival. A. The survival analysis in TCGA breast cancer cohort of ER+ and ER- patients shows that SERPINA1 has a significant predictive value only in the ER+ patients but not the ER- patients. B. Validation with ER+ and ER- patients in the Curtis breast cancer cohort confirms that SERPINA1 has a significant predictive value in the ER+ but not the ER- patients.

\section{Table 1: Summary of six patient cohorts tested for patient survival analyses.}

\begin{tabular}{|l|c|c|c|}
\hline Cohort & Total \# patients & \# of ER+ patients & \# of ER+ patients HER2 + / / Unknown \\
\hline TCGA & 779 & 570 & $88 / 318 / 164$ \\
\hline Curtis & 1284 & 986 & $77 / 909 / 0$ \\
\hline Bild & 170 & 114 & $61 / 40 / 13$ \\
\hline Chin & 117 & 74 & $4 / 45 / 25$ \\
\hline Desmedt & 198 & 134 & No HER2 status data \\
\hline Pawitan & 159 & 62 & $0 / 62 / 0$ \\
\hline Sotiriou & 99 & 65 & No HER2 status data \\
\hline
\end{tabular}

TCGA, Curtis and Bild cohorts have a significant number of HER2 positive patients. The other 4 cohorts have mostly HER2 negative or HER2 status unknown patients. 

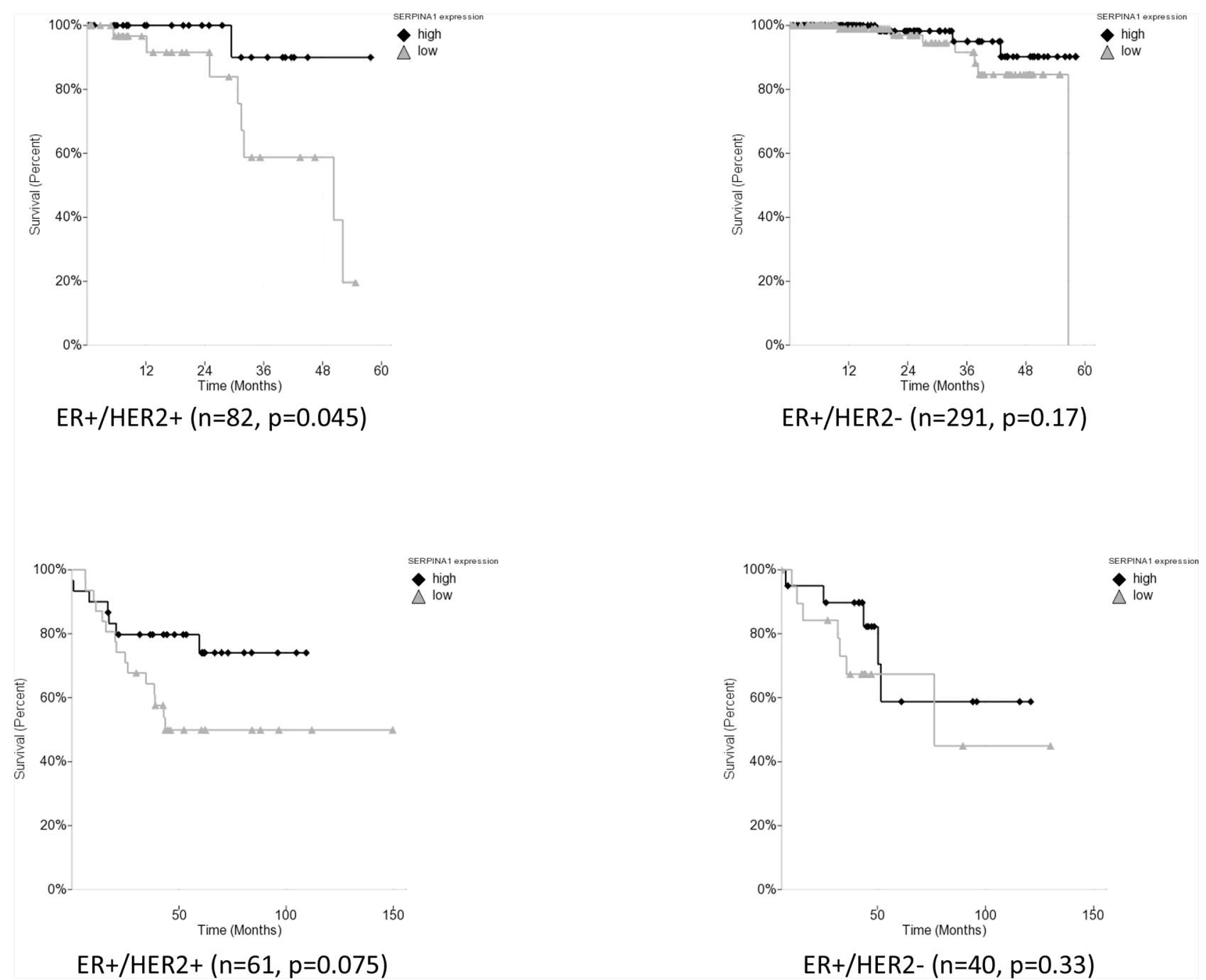

Figure 6: Survival analysis of SERPINA1 in TCGA and Bild breast cancer patient cohorts with ER+/HER2+ status. A. SERPINA1 has a significant predictive value in the ER+/HER2+ but not the ER+/HER2- patients from TCGA cohort with OS outcome. B. Validation with ER+/HER2+ and ER+/HER2- patients in the Bild cohort. DFS analysis of ER+/HER2+ patients in Bild patient cohort shows that patients with high expression of SERPINA1 has a better treatment outcome.

able to partially suppress the proliferation of LTEDaro [5]. However, several signal transduction pathways were found to be activated and crosstalk with ER in this AI-resistant line [5]. HER2 is one of the important signaling proteins that play a role in the phosphorylation of ER, as in luminal $B$ breast cancer [41]. In our laboratory, we generated an MCF-7 cell line that over-expresses aromatase and HER2, i.e., HER2-aro, and showed that this line was resistant to both AIs and ICI [5]. Addition of E2 to LTEDaro provided additional ER binding sites (data not shown). The physiological significance of E2 induced ER binding in LTEDaro E2 requires further molecular characterization.

Through the bioinformatics analysis in combination with gene expression microarray data, we identified SERPINA1 as one such ER target gene that clearly had E2-dependent ER binding in MCF-7aro cells and stronger E2-independent ER binding in LTEDaro cells. Its expression in both types of cells was significantly suppressed by the treatment of ER degrader, fulvestrant. Here we confirmed that SERPINA1 is a direct ER target gene, as supported by our ER ChIP-seq, ChIP PCR validation, microarray, gene expression qPCR data, and siRNA results. Considering the fact that SERPINA1 is highly expressed in LTEDaro, its expression must be upregulated by ER through cross-talk with growth factorregulated pathways. From searching the GEO data, we found that SERPINA1 is also a HER2-regulated gene. This was supported by the fact that HER2-aro cells had much higher expression levels of SERPINA1 than MCF-7aro cells. The expression of SERPINA1 in these resistant cells was reduced upon the treatment of HER2 siRNA (Figure 4B). Based on our results, we hypothesize that in LTEDaro and HER2-aro cells, ER is activated through phosphorylation by signaling pathways activated by HER2 [5] and SERPINA1 is a unique gene whose expression can be induced by phosphorylated ER. 
As a translational research project, this is a good example how we can correlate our ER binding data to patient outcome information using bioinformatics analysis. Our attempts pointed out some limitations in such effort. Our Kaplan Meier survival analysis found that SERPINA1 expression had a significant association with patient survival in ER+ patients using TCGA and Curtis cohorts, and with ER+/HER2+ patient survival in the TCGA and Bild cohorts. However, we were not able to reach the same conclusion using four other patient cohorts (i.e., Chin, Pawitan, Desmedt, Sotiriou). This could be due to differences in the cohort size and composition of patient population of ER/PR/HER2 status in these cohorts. This experience pointed out the need of large cohorts with detailed clincopathological features and treatment outcome information for more meaningful prediction. Since the majority of breast cancer patients are ER+, our finding that SERPINA1 can predict survival in ER+ patients suggests that it could be a potential prognostic marker, and many patients may benefit from this additional knowledge. Although the results were not expected originally, we are excited about the potential predictive value of SERPINA1 transcript expression levels in the ER+/HER2+ breast cancer, since these patients have relatively poor prognosis.

As two major regulatory pathways, ER and HER2 cross-talk when they co-exist $[42,43]$. Approximately 10 percent of breast cancer patients are ER+ and HER2+, and these patients have worse survival compared to ER+/ HER2- patients [44]. The ER+/HER2+ breast cancer is an important subtype of luminal B breast cancer [41]. It has been also observed that a significant number of recurring tumors from luminal A cancer are converted to luminal B HER2+ [41]. Overexpression of HER2 in ER+ breast cancer is well recognized to reduce the effectiveness of endocrine therapy, as observed preclinically [5] and clinically [45]. Similarly, co-expression of ER is known to result in a poor trastuzumab response [46]. Previous studies have shown that in HER2+ breast tumors, the mRNA levels of HER2 is correlated with pathological complete response (pCR) rate only in $\mathrm{ER}+$ patients but not in ER-patients [47]. Extensive studies have been performed to demonstrate how ER-regulated pathways and HER2-regulator pathways can modulate each other [43]. Preclinical experiments from our laboratory have found that LTEDaro, an AI-resistant model, is still partially responsive to ICI, but HER2-aro, a HER2-overexpressing line, fails to respond to either AI or ICI. These studies point out that in ER+/HER2+ cancer, it is essential to suppress both regulatory pathways, possibly additional mechanisms regulated both of these pathways.

While the expression level of SERPINA1 was found to be higher in endocrine resistant cells than responsive cells, unexpectedly, the Kaplan Meier survival analysis revealed that high expression of this gene associated with better survival in ER and HER2 positive luminal B subtype of breast cancer. To explain our findings, we hypothesize that a high expression of SERPINA1 indicates the important roles of ER and HER2 in driving the growth of the tumors. Therefore, in ER+ breast cancers, the expression of SERPINA1 could be an indication of estrogen-mediated ER activation and its expression levels correlate to the survival. A high expression of this gene is thought to be a strong indicator for the cooperative activation by both signaling pathways and to be a "good" response to both anti-ER and anti-HER2 therapies. Many ER-regulated genes, such as TFF1, PGR and GREB1, are known to be induced in endocrine-resistant cancer, but did not show statistical significant correlation with survival outcome based on our analysis.

In early stage breast cancer, women with ER+/ HER2+ cancers are treated with adjuvant trastuzumab. Recent data suggests that a subset of these patients may not benefit from trastuzumab [48]. Clinical data has shown that ER+/ HER2+ patients generally have worse outcome than ER-/HER2+ or ER+/HER2- patients [17, 18], and a predictive marker to predict a subgroup of patients with better outcome will be valuable. To investigate whether there are any differences in the treatments received by the patients with better survival compared to those with worse survival, we have examined the treatment information of ER+ and HER2+ patients in the TCGA cohort, but such information is difficult to obtain with treatment outcome. We made an attempt to compare the limited treatment information that we have of the patients with high and low levels of SERPINA1 expression, which corresponds to better and worse survival groups respectively (Supplementary Table 2). There were no major differences in the treatment strategies between good and bad responders, suggesting that SERPINA1 could be an outcome predictor independent of treatment options. Chemotherapy and HER2-directed therapy was widely used as systemic treatment for patients with ER+ and HER2+ disease. Anti-HER2 therapy has been shown to improve endocrine therapy in $\mathrm{ER}+/ \mathrm{HER} 2+$ positive cancer, as demonstrated in preclinical models [49, 50]. A recently completed trial revealed that a combination of anti-HER2 therapy and endocrine therapy could be valuable to treat ER+/HER2+ patients [51]. Furthermore, from the EGF30008 and TAnDEM (TrAstuzumab in Dual HER2 ER-positive Metastatic breast cancer) trials, lapatinib + letrozole and trastuzumb + anastrozole were shown to improve time to progression versus AI monotherapy, respectively [52]. A detailed analysis was reported by Delea et al. [52] that lapatinib + letrozole was not likely to be cost-effective than trastuzumab + anastrozole. Therefore, for those ER+/HER2+ patients with high levels of SERPINA1 expression, a less toxic or more cost-effective treatment may be also considered.

In conclusion, this is a translational research study. Attempts are made to translate results from ER ChIP-seq analysis to breast cancer patient outcome information. Ross-Innes et al. [35] have provided a strong precedence 
that differential ER-binding is associated with clinical outcome in breast cancer. Based on our findings, we propose that the expression of SERPINA1, an ER and HER2 regulated gene, is linked to the outcome of ER+ and ER+/HER2+ breast cancer.

\section{MATERIALS AND METHODS}

\section{Cell lines}

The MCF-7aro cell line was generated as a model to study the action of AIs [3]. The LTEDaro cell line was generated by a long-term estrogen deprivation of MCF-7aro and is used as a model of the late stage of endocrine resistance [4]. HER2-aro is a MCF-7 line that over-expresses HER2 and aromatase [5] and is a model of de novo AI resistance as well as a model of luminal $\mathrm{B}$, HER2-overexpressing subtype.

\section{ER ChIP-seq analysis}

MCF-7aro and LTEDaro cells were cultured in hormone-free MEM for 5 days and serum-free MEM for 1 day. When the cell number reached about $1 \times 10^{7}$, both the LTEDaro and MCF-7aro cell lines were serum starved for 24 hours followed by treatment with $100 \mathrm{nM}$ E2 or DMSO vehicle for 45 minutes, and cross-linked with 1\% formaldehyde at room temperature for 10 minutes. Cells were enlarged in hypotonic buffer and nuclei were isolated by addition of NP-40 and centrifugation. The chromatin was sonicated to yield a majority of fragments with sizes between 100-300 base-pairs (bp). ER $\alpha$ antibodies (HC-20; sc-543) and IgG antibodies (sc-2027) from Santa Cruz Biotechnologies (Santa Cruz, CA) were used for the immunoprecipitation and control respectively. The enriched chromatin was purified with the Qiagen Minelute PCR purification kit (Valencia, CA) and prepared for highthroughput sequencing.

The purified ChIP DNA samples were sequenced, using Illumina Solexa Genome Analyzer II (San Diego, CA) at the DNA sequencing core facility (City of Hope, Duarte, CA), to generate short reads that are 36 to $45 \mathrm{bp}$ in length. The short reads were mapped to human genome ( $\mathrm{Hg} 18)$ using the Bowtie [20] alignment tool. Peak-calling software, MACS v1.4.1 [21], was used to detect binding sites using the alignment results by setting a statistically significant cutoff ( $p$-value $=1.00 \mathrm{e}-5)$ comparing the ER versus IgG sample.

\section{SERPINA1 promoter cloning and luciferase activity assays}

The SERPINA1 promoter region was cloned into the pGL3 luciferase vector from Promega (Madison, WI). From the ChIP-seq peak calling data, the ER binding site covers a region of approximately $2 \mathrm{~kb}$ which overlaps with the TSS. The predicted ERE motif lies near the center of this binding region and is $19 \mathrm{bp}$ in length. We cloned a region that covers the TSS and upstream $2 \mathrm{~kb}$ region, with a total length of $2.1 \mathrm{~kb}$, which contains the full length SERPINA1 promoter. We also generated truncated versions of this promoter, with and without the predicted ERE motif, which are $270 \mathrm{bp}$ and $240 \mathrm{bp}$ in length respectively (Figure $3 \mathrm{~A}$ ). MCF-7aro and LTEDaro cells were then transfected using X-treme gene HP reagent (Roche, Indianapolis, IN) and assayed for luciferase activity.

\section{Overlap analysis of ER binding sites}

The ER binding sites from MCF-7aro E2 and LTEDaro DMSO were selected by FDR $\leq 0.5 \%$, and the sites were labeled as "common" if there was at least $1 \mathrm{bp}$ overlap, and the remainder sites were labeled as "unique". The ER binding sites associated with resistant cells were identified by comparing normalized binding site intensities of LTEDaro DMSO over MCF-7aro E2 with a change ratio $\geq 0.9$ as the cutoff. A positive fold change of $\geq 0.9$ represents LTEDaro DMSO binding sites that have $90 \%$ or greater binding intensity compared to MCF-7aro E2. This group of ER binding sites that are important in resistant cells was annotated with genes within $+/-20 \mathrm{~kb}$, and were then integrated with the gene expression data from our previous microarray study [1]. The genes were filtered based on a cutoff of 1.2 fold change with FDR adjusted $p<0.05$, and there were 350 genes that passed all filters.

\section{Kaplan-Meier survival analysis}

To identify genes with potential survival predictive power, the 350 genes from the ER-binding site overlap analysis were ranked based on Cox scores, which represents the association of gene expression in patient cohorts with patient survival data. For a single gene survival correlation, patients were grouped as high expression and low expression subgroups based on the median expression of that gene. For a group of genes, patients were grouped as High-Risk and Low-Risk subgroups based on 2-means clustering of the selected significant genes for Kaplan-Meier survival analysis [22]. Cox scores were calculated using R Bioconductor v3.0, and 2-means clustering analysis was performed in Partek Genomics Suite 6.6. Kaplan-Meier survival analysis was then used to determine the survival differences between the High-Risk and Low-Risk subgroups with $p$-values calculated by log-rank test in Partek Genomics Suite 6.6. Based on the study by Bair and Tibshirani, we used a Cox score cutoff of 2.39 to select top genes with better survival correlation [22], resulting in a list of 35 genes. Using TCGA breast cancer patient cohort as the training set [6], we performed further analysis and discovered a single gene, and validation was performed in the Curtis and Bild cohorts [23, 24]. In addition to the TCGA, Curtis and Bild cohorts, we have performed the survival analysis on four other patient cohorts, namely Chin, Desmedt, Pawitan, and Sotiriou [25-28]. 


\section{Semi-quantitative PCR analysis of ER binding}

ChIP DNA was prepared as described above. Primers were designed to amplify a 166bp region overlapping with the center of the peak as detected by ChIP-seq. The PCR was performed for 30 cycles using Promega GoTaq Green mastermix (Madison, WA) and analyzed by agarose gel electrophoresis.

\section{Quantitative PCR analysis of SERPINA1 expression}

For gene expression quantification, MCF-7aro LTEDaro, HER2-aro, BT-474 and MDA-MB-361 cells were treated for 24 hours with $1 \mathrm{nM} \mathrm{E} 2,200 \mathrm{nM}$ ICI 182780 , and/or HER2 siRNA. RNA was extracted from cells with Trizol reagent, and cDNA was synthesized with SuperScript III system from Invitrogen (Grand Island, NY). Quantification of cDNA was performed using the Bio-rad iQ5 system. For the gene expression analysis, the delta $\mathrm{Ct}$ method was used, with $\beta$-actin as the normalizer, and the gene expression values were calculated relative to the DMSO control. Primer sequences are provided in the Supplementary Table 1. SERPINA1 gene expression primer sequences have been previously published [8].

\section{ACKNOWLEDGMENTS}

We would like to thank Karineh Petrossian, Dr. Shang V. Wu and Dr. Yuanzhong Wang for their assistance in this study.

\section{SOURCES OF SUPPORT}

Financial support: This study was partially supported by the National Institutes of Health (NIH) grant R01 CA44735, National Cancer Institute of the NIH (P30CA33572), and the California Breast Cancer Research Program (CBCRP) Dissertation Award 15GB0027. Research reported in this publication included work performed in the Bioinformatics core and Interactive Genomics core supported by the National Cancer Institute of the NIH under award number P30CA33572.

\section{CONFLICTS OF INTEREST}

All authors declare no conflict of interest.

\section{REFERENCES}

1. Masri S, Phung S, Wang X, Wu X, Yuan YC, Wagman L, Chen S. Genome-wide analysis of aromatase inhibitor-resistant, tamoxifen-resistant, and long-term estrogen-deprived cells reveals a role for estrogen receptor. Cancer Res. 2008; 68:4910-4918.
2. Clarke R, Leonessa F, Welch JN, Skaar TC. Cellular and molecular pharmacology of antiestrogen action and resistance. Pharmacological reviews. 2001; 53:25-71.

3. Zhou DJ, Pompon D, Chen SA. Stable expression of human aromatase complementary DNA in mammalian cells: a useful system for aromatase inhibitor screening. Cancer Res. 1990; 50:6949-6954.

4. Chen S, Masri S, Hong Y, Wang X, Phung S, Yuan YC, $\mathrm{Wu} \mathrm{X}$. New experimental models for aromatase inhibitor resistance. J Steroid Biochem Mol Biol. 2007; 106:8-15.

5. Wong C, Wang X, Smith D, Reddy K, Chen S. AKT-aro and HER2-aro, models for de novo resistance to aromatase inhibitors; molecular characterization and inhibitor response studies. Breast Cancer Res Treat. 2012; 134:671-681.

6. Cancer Genome Atlas N. Comprehensive molecular portraits of human breast tumours. Nature. 2012; 490:61-70.

7. Finlay TH, Tamir S, Kadner SS, Cruz MR, Yavelow J, Levitz M. alpha 1-Antitrypsin- and anchorage-independent growth of MCF-7 breast cancer cells. Endocrinology. 1993; 133:996-1002.

8. Farshchian M, Kivisaari A, Ala-Aho R, Riihila P, Heljasvaara R, Kahari VM. Serpin peptidase inhibitor clade A member 1 (SerpinA1) is a novel biomarker for progression of cutaneous squamous cell carcinoma. The American journal of pathology. 2011; 179:1110-1119.

9. Tan XF, Wu SS, Li SP, Chen Z, Chen F. Alpha-1 antitryp$\sin$ is a potential biomarker for hepatitis B. Virology journal. $2011 ; 8: 274$.

10. de Sa SV, Correa-Giannella ML, Machado MC, Krogh K, de Almeida MQ, Albergaria Pereira MA, Coelho Siqueira SA, Patzina RA, Ibuki FS, Sogayar MC, Machado MC, Giannella-Neto D. Serpin peptidase inhibitor clade A member 1 as a potential marker for malignancy in insulinomas. Clin Cancer Res. 2007; 13:5322-5330.

11. Zhao W, Yang Z, Liu X, Tian Q, Lv Y, Liang Y, Li C, Gao X, Chen L. Identification of alpha1-antitrypsin as a potential prognostic biomarker for advanced nonsmall cell lung cancer treated with epidermal growth factor receptor tyrosine kinase inhibitors by proteomic analysis. The Journal of international medical research. 2013; 41:573-583.

12. Vierlinger K, Mansfeld MH, Koperek O, Nohammer C, Kaserer K, Leisch F. Identification of SERPINA1 as single marker for papillary thyroid carcinoma through microarray meta analysis and quantification of its discriminatory power in independent validation. BMC medical genomics. 2011; 4:30.

13. Topic A, Ljujic M, Nikolic A, Petrovic-Stanojevic N, Dopudja-Pantic V, Mitic-Milikic M, Radojkovic D. Alpha1-antitrypsin phenotypes and neutrophil elastase gene promoter polymorphisms in lung cancer. Pathology oncology research : POR. 2011; 17:75-80.

14. Abbott KL, Aoki K, Lim JM, Porterfield M, Johnson R, O’Regan RM, Wells L, Tiemeyer M, Pierce M. Targeted glycoproteomic identification of biomarkers for human 
breast carcinoma. Journal of proteome research. 2008; 7:1470-1480.

15. Doustjalali SR, Yusof R, Yip CH, Looi LM, Pillay B, Hashim $\mathrm{OH}$. Aberrant expression of acute-phase reactant proteins in sera and breast lesions of patients with malignant and benign breast tumors. Electrophoresis. 2004; 25:2392-2401.

16. Lopez-Arias E, Aguilar-Lemarroy A, Felipe Jave-Suarez L, Morgan-Villela G, Mariscal-Ramirez I, MartinezVelazquez M, Alvarez AH, Gutierrez-Ortega A, HernandezGutierrez R. Alpha 1-antitrypsin: a novel tumor-associated antigen identified in patients with early-stage breast cancer. Electrophoresis. 2012; 33:2130-2137.

17. Dawood S, Broglio K, Buzdar AU, Hortobagyi GN, Giordano SH. Prognosis of women with metastatic breast cancer by HER 2 status and trastuzumab treatment: an institutional-based review. J Clin Oncol. 2010; 28:92-98.

18. Jerjees DA, Alabdullah M, Green AR, Alshareeda A, Macmillan RD, Ellis IO, Rakha EA. Prognostic and biological significance of proliferation and HER2 expression in the luminal class of breast cancer. Breast Cancer Res Treat. 2014; 145:317-30.

19. Finn RS, Press MF, Dering J, O'Rourke L, Florance A, Ellis C, Martin AM, Johnston S. Quantitative ER and PgR assessment as predictors of benefit from lapatinib in postmenopausal women with hormone receptor-positive, HER2negative metastatic breast cancer. Clin Cancer Res. 2014; 20:736-743.

20. Langmead B, Trapnell C, Pop M, Salzberg SL. Ultrafast and memory-efficient alignment of short DNA sequences to the human genome. Genome Biol. 2009; 10:R25.

21. Zhang Y, Liu T, Meyer CA, Eeckhoute J, Johnson DS, Bernstein BE, Nusbaum C, Myers RM, Brown M, Li W, Liu XS. Model-based analysis of ChIP-Seq (MACS). Genome Biol. 2008; 9:R137.

22. Bair E, Tibshirani R. Semi-supervised methods to predict patient survival from gene expression data. PLoS biology. 2004; 2:E108.

23. Curtis C, Shah SP, Chin SF, Turashvili G, Rueda OM, Dunning MJ, Speed D, Lynch AG, Samarajiwa S, Yuan Y, Graf S, Ha G, Haffari G, Bashashati A, Russell R, McKinney S, et al. The genomic and transcriptomic architecture of 2, 000 breast tumours reveals novel subgroups. Nature. 2012; 486:346-352.

24. Bild AH, Yao G, Chang JT, Wang Q, Potti A, Chasse D, Joshi MB, Harpole D, Lancaster JM, Berchuck A, Olson JA Jr, Marks JR, Dressman HK, West M, Nevins JR. Oncogenic pathway signatures in human cancers as a guide to targeted therapies. Nature. 2006; 439:353-357.

25. Chin K, DeVries S, Fridlyand J, Spellman PT, Roydasgupta R, Kuo WL, Lapuk A, Neve RM, Qian Z, Ryder T, Chen F, Feiler H, Tokuyasu T, Kingsley C, Dairkee S, Meng Z, et al. Genomic and transcriptional aberrations linked to breast cancer pathophysiologies. Cancer cell. 2006; 10:529-541.
26. Desmedt C, Piette F, Loi S, Wang Y, Lallemand F, HaibeKains B, Viale G, Delorenzi M, Zhang Y, d'Assignies MS, Bergh J, Lidereau R, Ellis P, Harris AL, Klijn JG, Foekens JA, et al. Strong time dependence of the 76-gene prognostic signature for node-negative breast cancer patients in the TRANSBIG multicenter independent validation series. Clin Cancer Res. 2007; 13:3207-3214.

27. Pawitan Y, Bjohle J, Amler L, Borg AL, Egyhazi S, Hall P, Han X, Holmberg L, Huang F, Klaar S, Liu ET, Miller L, Nordgren H, Ploner A, Sandelin K, Shaw PM, et al. Gene expression profiling spares early breast cancer patients from adjuvant therapy: derived and validated in two population-based cohorts. Breast cancer research : BCR. 2005; 7:R953-964.

28. Sotiriou C, Neo SY, McShane LM, Korn EL, Long PM, Jazaeri A, Martiat P, Fox SB, Harris AL, Liu ET. Breast cancer classification and prognosis based on gene expression profiles from a population-based study. Proc Natl Acad Sci U S A. 2003; 100:10393-10398.

29. Simpson NE, Gertz J, Imberg K, Myers RM, Garabedian MJ. Research resource: enhanced genome-wide occupancy of estrogen receptor alpha by the cochaperone p23 in breast cancer cells. Mol Endocrinol. 2012; 26:194-202.

30. Lin CY, Vega VB, Thomsen JS, Zhang T, Kong SL, Xie M, Chiu KP, Lipovich L, Barnett DH, Stossi F, Yeo A, George J, Kuznetsov VA, Lee YK, Charn TH, Palanisamy N, et al. Whole-genome cartography of estrogen receptor alpha binding sites. PLoS genetics. 2007; 3:e87.

31. Moggs JG, Murphy TC, Lim FL, Moore DJ, Stuckey R, Antrobus K, Kimber I, Orphanides G. Anti-proliferative effect of estrogen in breast cancer cells that re-express ERalpha is mediated by aberrant regulation of cell cycle genes. J Mol Endocrinol. 2005; 34:535-551.

32. Creighton CJ, Hilger AM, Murthy S, Rae JM, Chinnaiyan AM, El-Ashry D. Activation of mitogenactivated protein kinase in estrogen receptor alpha-positive breast cancer cells in vitro induces an in vivo molecular phenotype of estrogen receptor alpha-negative human breast tumors. Cancer Res. 2006; 66:3903-3911.

33. Chen Z, Yuan YC, Wang Y, Liu Z, Chan HJ, Chen S. Down-regulation of programmed cell death 4 (PDCD4) is associated with aromatase inhibitor resistance and a poor prognosis in estrogen receptor-positive breast cancer. Breast Cancer Res Treat. 2015; 152:29-39.

34. Manavathi B, Dey O, Gajulapalli VN, Bhatia RS, Bugide S, Kumar R. Derailed estrogen signaling and breast cancer: an authentic couple. Endocrine reviews. 2013; 34:1-32.

35. Ross-Innes CS, Stark R, Teschendorff AE, Holmes KA, Ali HR, Dunning MJ, Brown GD, Gojis O, Ellis IO, Green AR, Ali S, Chin SF, Palmieri C, Caldas C, Carroll JS. Differential oestrogen receptor binding is associated with clinical outcome in breast cancer. Nature. 2012; 481:389-393.

36. Carroll JS, Meyer CA, Song J, Li W, Geistlinger TR, Eeckhoute J, Brodsky AS, Keeton EK, Fertuck KC, 
Hall GF, Wang Q, Bekiranov S, Sementchenko V, Fox EA, Silver PA, Gingeras TR, et al. Genome-wide analysis of estrogen receptor binding sites. Nat Genet. 2006; 38:1289-1297.

37. Kininis M, Chen BS, Diehl AG, Isaacs GD, Zhang T, Siepel AC, Clark AG, Kraus WL. Genomic analyses of transcription factor binding, histone acetylation, and gene expression reveal mechanistically distinct classes of estrogen-regulated promoters. Mol Cell Biol. 2007; 27:5090-5104.

38. Kwon YS, Garcia-Bassets I, Hutt KR, Cheng CS, Jin M, Liu D, Benner C, Wang D, Ye Z, Bibikova M, Fan JB, Duan L, Glass CK, Rosenfeld MG, Fu XD. Sensitive ChIPDSL technology reveals an extensive estrogen receptor alpha-binding program on human gene promoters. Proc Natl Acad Sci U S A. 2007; 104:4852-4857.

39. Liu Y, Gao H, Marstrand TT, Strom A, Valen E, Sandelin A, Gustafsson JA, Dahlman-Wright K. The genome landscape of ERalpha- and ERbeta-binding DNA regions. Proc Natl Acad Sci U S A. 2008; 105:2604-2609.

40. Wong C, Chen S. The development, application and limitations of breast cancer cell lines to study tamoxifen and aromatase inhibitor resistance. J Steroid Biochem Mol Biol. 2012; 131:83-92.

41. Falck AK, Bendahl PO, Chebil G, Olsson H, Ferno M, Ryden L. Biomarker expression and St Gallen molecular subtype classification in primary tumours, synchronous lymph node metastases and asynchronous relapses in primary breast cancer patients with 10 years' follow-up. Breast Cancer Res Treat. 2013; 140:93-104.

42. Takada M, Higuchi T, Tozuka K, Takei H, Haruta M, Watanabe J, Kasai F, Inoue K, Kurosumi M, Miyazaki M, Sato-Otsubo A, Ogawa S, Kaneko Y. Alterations of the genes involved in the PI3K and estrogen-receptor pathways influence outcome in human epidermal growth factor receptor 2-positive and hormone receptor-positive breast cancer patients treated with trastuzumab-containing neoadjuvant chemotherapy. BMC Cancer. 2013; 13:241.

43. Giuliano M, Trivedi MV, Schiff R. Bidirectional Crosstalk between the Estrogen Receptor and Human Epidermal Growth Factor Receptor 2 Signaling Pathways in Breast Cancer: Molecular Basis and Clinical Implications. Breast Care (Basel). 2013; 8:256-262.

44. Howlader N, Altekruse SF, Li CI, Chen VW, Clarke CA, Ries LA, Cronin KA. US Incidence of Breast Cancer Subtypes Defined by Joint Hormone Receptor and HER2 Status. J Natl Cancer Inst. 2014; 106(5).
45. Collins LC, Schnitt SJ. HER2 protein overexpression in estrogen receptor-positive ductal carcinoma in situ of the breast: frequency and implications for tamoxifen therapy. Modern pathology : an official journal of the United States and Canadian Academy of Pathology, Inc. 2005; 18:615-620.

46. Collins DC, Cocchiglia S, Tibbitts P, Solon G, Bane FT, McBryan J, Treumann A, Eustace A, Hennessy B, Hill AD, Young LS. Growth factor receptor/steroid receptor cross talk in trastuzumab-treated breast cancer. Oncogene. 2015; 34:525-30.

47. Denkert C, Huober J, Loibl S, Prinzler J, Kronenwett R, Darb-Esfahani S, Brase JC, Solbach C, Mehta K, Fasching PA, Sinn BV, Engels K, Reinisch M, Hansmann ML, Tesch H, von Minckwitz G, et al. HER2 and ESR1 mRNA expression levels and response to neoadjuvant trastuzumab plus chemotherapy in patients with primary breast cancer. Breast cancer research : BCR. 2013; 15:R11.

48. Pogue-Geile KL, Kim C, Jeong JH, Tanaka N, Bandos H, Gavin PG, Fumagalli D, Goldstein LC, Sneige N, Burandt E, Taniyama Y, Bohn OL, Lee A, Kim SI, Reilly ML, Remillard MY, et al. Predicting degree of benefit from adjuvant trastuzumab in NSABP trial B-31. J Natl Cancer Inst. 2013; 105:1782-1788.

49. Sabnis G, Schayowitz A, Goloubeva O, Macedo L, Brodie A. Trastuzumab reverses letrozole resistance and amplifies the sensitivity of breast cancer cells to estrogen. Cancer Res. 2009; 69:1416-1428.

50. Chen B, Wang Y, Kane SE, Chen S. Improvement of sensitivity to tamoxifen in estrogen receptor-positive and Herceptin-resistant breast cancer cells. J Mol Endocrinol. 2008; 41:367-377.

51. Rimawi MF, Mayer IA, Forero A, Nanda R, Goetz MP, Rodriguez AA, Pavlick AC, Wang T, Hilsenbeck SG, Gutierrez C, Schiff R, Osborne CK, Chang JC. Multicenter phase II study of neoadjuvant lapatinib and trastuzumab with hormonal therapy and without chemotherapy in patients with human epidermal growth factor receptor 2-overexpressing breast cancer: TBCRC 006. J Clin Oncol. 2013; 31:1726-1731.

52. Delea TE, Hawkes C, Amonkar MM, Lykopoulos K, Johnston SR. Cost-Effectiveness of Lapatinib plus Letrozole in Post-Menopausal Women with Hormone Receptor-and HER2-Positive Metastatic Breast Cancer. Breast Care (Basel). 2013; 8:429-437. 